\title{
Left atrium segmentation for atrial fibrillation ablation
}

\author{
R. Karim, R. Mohiaddin, D. Rueckert \\ Department of Computing, Imperial College London, London, UK \\ National Heart and Lung Institute, Imperial College London, London, UK
}

\begin{abstract}
Segmentation of the left atrium is vital for pre-operative assessment of its anatomy in radio-frequency catheter ablation (RFCA) surgery. RFCA is commonly used for treating atrial fibrillation. In this paper we present an semi-automatic approach for segmenting the left atrium and the pulmonary veins from MR angiography (MRA) data sets. We also present an automatic approach for further subdividing the segmented atrium into the atrium body and the pulmonary veins. The segmentation algorithm is based on the notion that in MRA the atrium becomes connected to surrounding structures via partial volume affected voxels and narrow vessels, the atrium can be separated if these regions are characterized and identified. The blood pool, obtained by subtracting the pre- and post-contrast scans, is first segmented using a regiongrowing approach. The segmented blood pool is then subdivided into disjoint subdivisions based on its Euclidean distance transform. These subdivisions are then merged automatically starting from a seed point and stopping at points where the atrium leaks into a neighbouring structure. The resulting merged subdivisions produce the segmented atrium. Measuring the size of the pulmonary vein ostium is vital for selecting the optimal Lasso catheter diameter. We present a second technique for automatically identifying the atrium body from segmented left atrium images. The separating surface between the atrium body and the pulmonary veins gives the ostia locations and can play an important role in measuring their diameters. The technique relies on evolving interfaces modelled using level sets. Results have been presented on 20 patient MRA datasets.
\end{abstract}

Keywords: Medical Image Segmentation, Visualization, MRI, Image-guided procedures, Atrial Fibrillation, Radio-frequency Catheter Ablation.

\section{INTRODUCTION}

One of the most common causes of deaths in patients with cardiovascular related illnesses are heart strokes and attacks. The American Heart Association reports that $15 \%$ of all heart strokes are caused by a lifethreatening condition called atrial fibrillation (AF) [1]. AF is a condition involving the left atrium of the heart. The left atrium is one of the four chambers of the heart. It receives oxygenated blood from the lungs and pumps it into the left ventricle. This blood is then circulated to the rest of the body. In a healthy adult the left atrium pumps blood into the ventricle in a regular rhythm. In AF, the left atrium quivers in an abnormal rhythm and is no longer able to pump blood into the left ventricle efficiently. This may cause possible pooling and clotting of blood in the left atrium which can lead to a stroke. Radio-frequency catheter ablation (RFCA) has become the treatment of choice for patients suffering from AF [2]. The objective of the ablation procedure is to eliminate sources of ectopic foci by charring tissues with high radio-frequency energy. These sources are identified pre-procedurally by examining electroanatomical maps that correlate the eletrophysiological characteristics with the cardiac anatomy. Medical literature indicates pulmonary venous drainages of the left atrium to be strong sources of ectopic focal activity [8]. These sources along with the atrium's anatomy are identified and examined pre-procedurally. Several measurements such as pulmonary vein (PV) ostia diameters are also taken prior to the procedure. An automatic segmentation of the left atrium and its pulmonary veins from MRA can give a unique nonobstructive view of the atrium's anatomy and assist greatly in pre-planning an image-guided intervention. In addition to this, a further decomposition of the segmented atrium into the atrial body and pulmonary veins (PV) can provide automatic recognition of locations of the ostia and assist in computing their diameters. A variety of complications of AF ablation have been reported [10], with some of them fatal in patients with structurally normal hearts. Major complications from AF ablation include vascular access accidents which can be reduced if the left atrial anatomy is thoroughly examined pre-procedurally. 


\section{RELATED WORK}

Relatively little work has been reported on automated left atrium segmentation. Berg et. al. [4] uses a shape-constrained deformable surface model to segment the left atrium from multi-slice CT images. However, the model is restricted to a mean surface model which is built from training samples. Although such a technique may perform well on the commonly occurring variants of the atrium, the underrepresentation of the rarely occurring variants in the mean model makes it difficult for it to fit to these instances. John et. al. [5] uses a data-driven approach for segmenting the atrium from MR Angiography (MRA) datasets. The segmented blood pool is subdivided into regions which are later merged but separated at narrowings. This technique is based on the notion that the atrium is commonly connected to neighbouring structures through a narrowing. Although the method is fast and robust, it suffers from several limitations such as its inability to segment in cases where there is a non-narrow connection between a pulmonary vein and the pulmonary artery caused by a string of partial volume affected voxels. Our method is similar to the one described in [5]. An important difference is the manner in which our system can correct for over and under-segmentations using very little user-interaction. A second difference is the calculation of saddle points in [5] which is replaced with finding a point with the highest Euclidean distance value in the separating surface between any two adjacent subdivisions. This produces a more accurate estimate of the true radius of the separating surface. A third difference is the use of automatic threshold selection for segmenting the blood pool which has greatly improved the quality of the results. We also introduce a new method for segmenting the atrial body using level set methods.

\section{LEFT ATRIUM SEGMENTATION}

The left atrium is a highly anatomically variable structure where the number and sizes of the pulmonary venous drainages can vary significantly across patients. Variations to the right and left drainage patterns of the atrium have been documented in [3]. This high degree of anatomic variability makes the left atrium segmentation problem non-trivial.

\subsection{Segmenting the blood pool}

The images acquired for this study are MRA images. Prior to segmentation, unwanted structures such as bones are easily removed by subtracting the post- from the pre-MRA scans. A rigid registration may be necessary at this stage allowing for the fact that the patient might have moved during image acquisition. The input to our segmentation system is thus the subtracted image showing only the enhanced vessels. The left atrium is surrounded by neighboring structures such as the pulmonary arteries, ascending aorta and the left ventricle. During acquisition, blood which is contrast-enhanced perfuse into these regions very quickly making it difficult to avoid imaging these neighboring structures. These neighbors are partly removed by using a bounding box which selects a region of interest around the left atrium, thereby excluding them as much as possible. The blood pool within the bounding box is extracted using a region-growing segmentation technique where a seed point inside the atrium is selected. The lower and upper thresholds for blood are automatically computed using the Otsu method [6]. In this method an optimal threshold value which maximizes the between-class variance between blood and non-blood tissue classes is determined. The output at this stage is a binary image with the segmented blood pool that contains the left atrium, its pulmonary venous drainages and segments of neighbouring vessels and structures. Although it is not vital to remove the ventricle blood pool in the context of RFCA, the bounding box can cut off the atrium from the ventricle blood pool along a suitable user-defined plane.

\subsection{Computing subdivisions}

The blood pool is subdivided into regions using a scheme described below. Neighbouring subdivisions are later merged automatically to produce the segmented atrium. The process of subdividing the blood pool is performed in three steps: 1) Computing the Euclidean distance transformation of the image. 2) Finding local maxima on the EDT map. 3) Determining subdivision membership for each voxel in image.

In the first step we compute the Euclidean distance transformation (EDT) of the binary image, where any voxel inside the blood pool has a value of 1 and any voxel outside has a value of 0 . A distance transform of 
an image $I$ is an assignment to each voxel $\mathrm{v}$ the distance between $\mathrm{v}$ and the closest feature voxel (CFV). The distance is computed using a defined distance metric. An EDT is one such class of distance transforms where the distance metric is defined to be the Euclidean distance between a pair of voxels. The CFV in our binary image are conveniently defined to be the edge voxels. The EDT for the binary image can be computed using a linear time algorithm [7]. Under the EDT transformation, a voxel deep inside the blood pool is assigned a high distance value whereas a voxel in the proximity of a boundary is assigned a relatively smaller value. Voxels outside the blood pool are assigned a distance value of zero. The next step is to compute the local maximum points on the EDT map. We use a 26-voxel neighborhood, for calculating local maxima. The local maximum is the point with the largest EDT in the defined neighborhood. After computing local maxima, for each voxel we compute the subdivision it belongs to. Starting from a voxel we search along the path of the maximum ascent until we reach a local maximum. The voxel belongs to the subdivision that is centered by this local maximum. In this way each voxel converges to a local maximum, and each local maximum produces unique subdivisions containing voxels which converge to it. Subdivisions are identified by their centers which is a local maximum. This is analogous to a Voronoi diagram where a Voronoi cell is similar to a subdivision. Figure 1(ii) shows how the entire blood pool is subdivided using the subdivision scheme described above.

\subsection{Automatic subdivision merging}

Once subdivisions are produced, they are selectively merged using a pre-defined criterion. They can also be manually merged by the user interactively selecting each individual subdivision which lie inside the atrium. For an automatic approach, a criterion is developed which exploits the anatomy: physically the left atrium is only connected to the left ventricle through the mitral valve. However, in MRA images, due to the partial volume effect the blood pool in the atrium becomes frequently connected to the blood in the ascending aorta. Additionally, the pulmonary veins also appear connected to the pulmonary artery. Any left atrium segmentation scheme must be robust against such artifacts. It can be safely assumed that these connections are very narrow regions connecting either sides of a blood pool. The subdivision scheme described in the previous section will produce subdivisions on either side of such narrowings. It now merely remains to describe a merging scheme which merges subdivisions not separated by such narrowings and stops merging subdivisions that are separated by a narrowing.

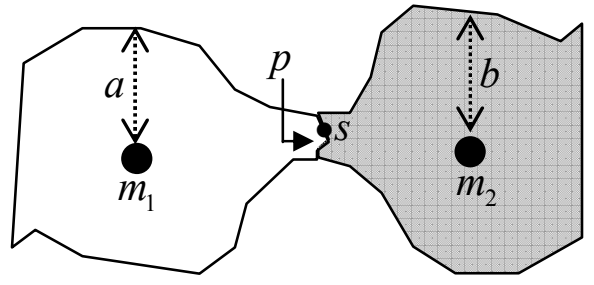

(i)

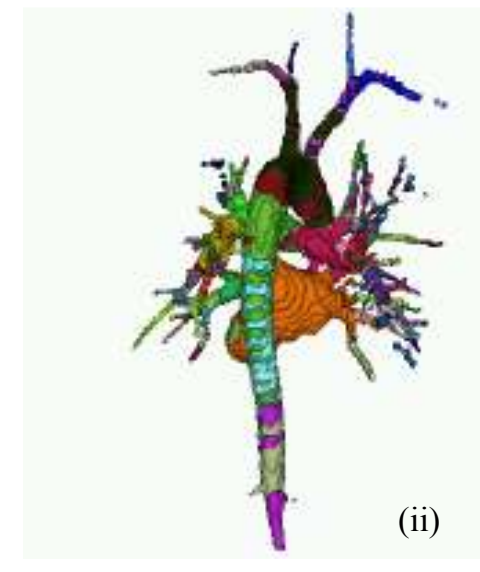

Figure 1: (i) Two subdivisions with their diameters and separating surface (ii) Subdivisions within a blood pool. Each subdivision is marked with a random color.

Consider the radiuses of two neighboring subdivisions and which are separated by a separating surface $\mathrm{s}$ (figure 1(i)). The radius of a subdivision is the Euclidean distance value of its local maximum. Assuming that $\mathrm{a}$ and $\mathrm{b}$ are the radiuses of two neighboring subdivisions with the separating surface radius denoted by p. A merging criterion can be described in terms of a merging value $T$ which is defined as:

$$
T=\min (a, b)-p
$$


The merging value can be controlled using a user-defined threshold. The merging criterion allows any two adjacent subdivisions to be merged when their merging value satisfy a threshold. It prevents merging of subdivisions where $T$ falls below the threshold. In this way, the radius of the separating surface at a narrowing will have a smaller radius compared to the radiuses of the adjacent subdivisions, thus yielding a large merging value. On the contrary, at non-narrow connections, the radius of the separating surface will be comparable to that of its adjacent subdivisions, thus giving a smaller merging value. Selecting an appropriate threshold value will thus stop the merging process at possible narrowings. The merging process starts from a user-selected seed point, preferably located close to the centre of the atrium. The subdivision containing the seed point is our seed subdivision. Similar to a region-growing approach, all subdivisions connected to the seed subdivision are merged on the condition that they fulfill the merging criterion. Symbolically, merging two subdivisions is equivalent to replacing them with a single subdivision with a radius equal to the larger of the two radiuses. At the end of the merging process the resulting merged subdivisions is the segmented atrium separated from the rest of the connected structures.

\section{ATRIAL BODY SEGMENTATION}

In image-guided RFCA it would be ideal if a patient's left atrium anatomy can be automatically classified. Further quantifying ostium diameters can help select the right catheter size. Solving this problem computationally would require an automatic labeling scheme for atrium's primary components, i.e. the atrial body and the pulmonary veins. Thus, a preliminary step of this scheme is atrial body segmentation. We also envisage that the detection of pulmonary veins can be simplified, and the search space reduced if the atrial body can be located and segmented.

The method is based on evolving surfaces, which can either be explicit such as region growing or active contours, or implicit such as geodesic active contours or level set methods. Here, we employ level set methods for automatic left atrial body segmentation. The idea is to use a propagating front to stop at the atrial body edges, except in places where the atrial body meets the pulmonary veins where it is stopped using the combined effects of the speed image and a curvature constraint. In a level set method framework, the evolving surface is implicitly defined as the zero level set of a higher dimensional distance or level set function $\phi$. Consider an evolving surface with position denoted by $\vec{S}$ and a speed function $F$ which gives the speed of $\vec{S}$ in its normal direction. The surface $\vec{S}$ is embedded in a higher dimensional function $\phi$, the zero level set of which is given by the equation:

$$
\phi(\vec{S}, t)=0
$$

Then, by the chain rule, an evolution equation for the evolving surface is obtained:

$$
\phi_{t}+F|\nabla \phi|=0
$$

This is an initial value partial differential equation of the Hamilton-Jacobi type which can be solved using various numerical schemes [13]. The choice of speed function $F$ is crucial for segmentation as it determines the convergence speed and the accuracy of the final atrial boundary positions. A simple speed function can be constructed which stops the level set at object boundaries:

$$
F(x, y, z)=e^{-\left|\nabla G_{\sigma} * I(x, y, z)\right|}
$$

Here $G_{\sigma} * I(x, y, z)$ is the convolution of the image with a Gaussian smoothing filter with a characteristic width $\sigma$. However, this is not adequate as an unsupervised propagating front can easily diffuse into the PV drainages connected to the atrial body. Our choice of the speed function restricts the level set to remain within the atrial body and stops it from propagating further into PV. According to [12] the speed function can be split into two components: 


$$
F=F_{A}+F_{G}
$$

where $F_{A}$ is the advection term and is independent of the propagating front's geometry. The second term $F_{G}$ depends on the geometry of the front, such as its local curvature. Equation 5 under the influence of $F_{A}$ and $F_{G}$ now becomes:

$$
\phi_{t}+F_{A}|\nabla \phi|+F_{G}|\nabla \phi|=0
$$

We use the Euclidean distance transform function as the advection speed term. It produces a reasonable speed function which can cause the propagating front to grow within the atrial body (high EDT value) and stop near the boundary of the structure (an EDT value of 0 at the boundary). It also causes the front's speed to decelerate at the mouth of a PV (i.e. ostium) where at such locations it may encounter small values of EDT due to a narrowing. However, the EDT alone is not sufficient enough to stop the front from propagating into $\mathrm{PV}$. To this end we use the second speed term $F_{G}$ in Equation 5 to impose a curvature constraint on the front's geometry. The curvature is computed using:

$$
\kappa=\nabla \cdot \frac{\nabla \phi}{|\nabla \phi|}
$$

A speed term which moves proportional to the front's curvature can now be defined, where $\varepsilon$ is the curvature constraint control parameter:

$$
F_{G}=1-\varepsilon \kappa
$$

Thus, the propagating front driven by the level set equation 6 with the above mentioned speed functions can segment the atrial body but only reasonably well; we observed that the front stopped prematurely close to the atrial body wall. This is caused by the EDT-driven advection term which approaches values close to zero near the atrial boundary. However, this first step is yet important since we acquire a good approximation of the atrial body's shape. The segmentation is next further improved by pipelining it into a second level set scheme. The segmentation obtained in the previous step is used as an initial contour in this second step. The propagating front uses a simple image gradient based advection term such as in equation 4 together with a relatively high curvature constraint. These two factors cause the front to restrict itself to an initial shape and to propagate further towards atrial boundaries. Note that although the advection term allows the front to leak into the PV this time, it is yet prevented to do so because of the high curvature constraint.

\section{RESULTS}

\subsection{Segmentation results of the left atrium}

We have tested our left atrium segmentation technique on 20 patient MRA datasets. The datasets acquired were diverse in terms of the anatomy of the left atrium and its pulmonary veins. One of the datasets had an abnormal enlarging of the left atrial body (figure 2a), and one of them had an unusual left drainage where the two left pulmonary veins joined into one vessel (figure $2 \mathrm{c}$ ). The proposed technique was robust against these cases and all cases were segmented successfully. The segmentation results were evaluated by an expert clinician by overlaying the segmentation on the original MRI and noting the differences. An important criterion for a good left atrial segmentation for RFCA is that it must include all the pulmonary veins to the left atrium. Missing a pulmonary vein can lead to an unsuccessful RFCA procedure where the patient can complain of recurring episodes of AF caused by the overlooked pulmonary vein [9]. The inclusion of all pulmonary veins in the segmentation was thus easily validated using this overlaying technique. 
Once the EDT and subdivisions are computed, the segmentation process is almost instant taking less than a second for each MRA image. Computation of the EDT for the image is also very fast taking less than a second. However, the subdivision computation can take a few seconds depending on the size of the dataset.

\subsection{Segmentation results of the atrial body}

We tested our atrial body segmentation technique on the segmented atriums. Figure 3 shows some of the results where the segmented atrial body (in white) has been overlaid on top of the segmented atrium (in grey). The images shown here are only single-slice images. It is clear from these images that the propagating front does not leak into the PV. However, in some cases it may not perfectly segment and trace out the atrial wall boundary due to the high curvature constraint imposed on the level set. We feel in the context of image-guided RFCA this may not be as crucial as is to detect the number and locations of PV and ostium, of which atrial body segmentation is an important preliminary step. Figure 3 shows an endocardial view of the segmented atrium with the ostium clearly visible. Electrophysiologists sometime also prefer an endocardial view where a clear view of the PV drainage openings are visible. This view is important image-guided RFCA allowing the circumferential path of ablation to be traced easily
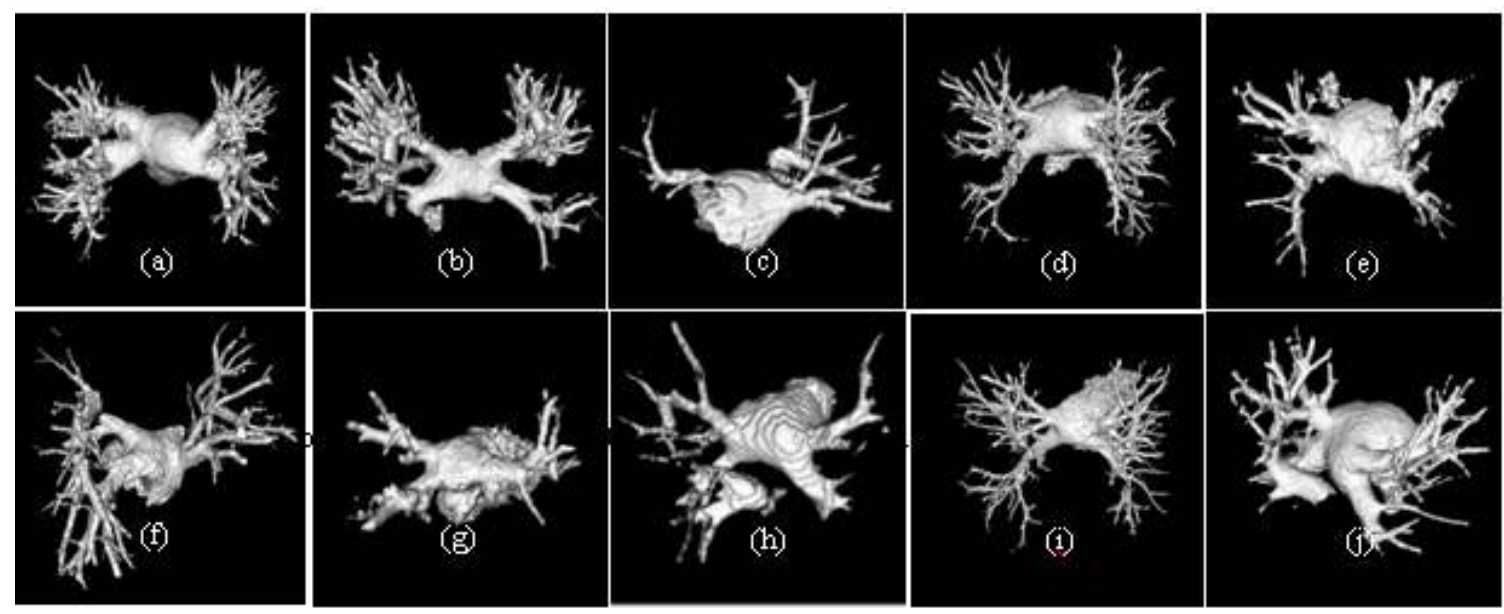

Figure 2: Marching cubes iso-surface reconstruction of the segmented left atriums of 10 patient MRA datasets. Note the variation in shape, size and anatomy of the atrium across patients.
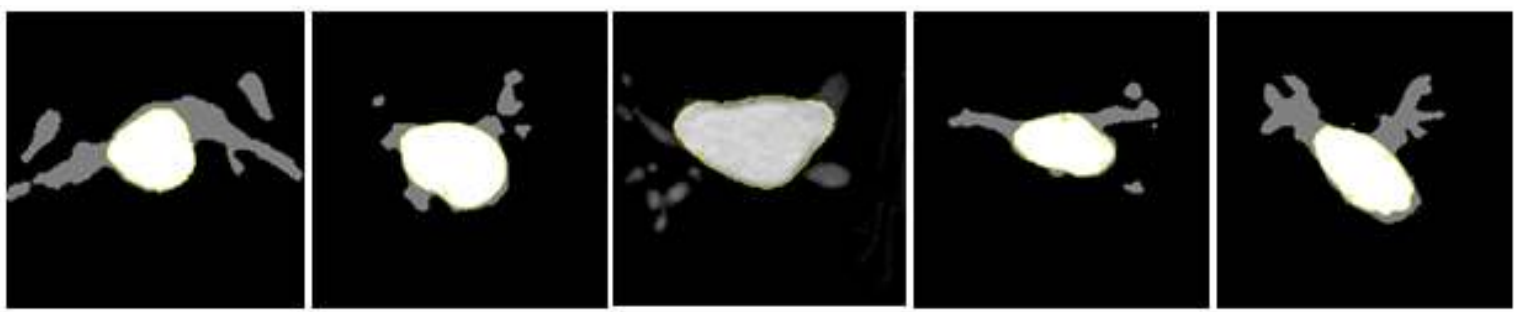

Figure 3: Single slice images of the segmented atrial body (white) overlaid on top of the segmented atrium (grey).
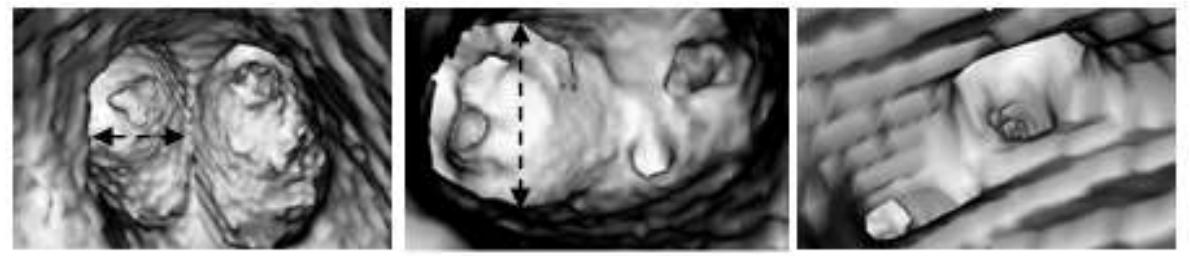

Figure 4: Endocardial views of the segmented atriums for measuring ostia diameters 


\section{DISCUSSION}

Due to the high degree of anatomic variability in the left atrium un-predictable shape variations may occur and can make such a data-driven technique susceptible to mis-segmentation. In at least one of the patient data we have noticed narrow regions within the atrium (figure 2a). This causes the algorithm to cut part of the atrium off at the narrowing producing an under-segmented left atrium. If these narrowings are within an opening of a drainage, an entire pulmonary drainage will be excluded from the segmentation leading to possible surgical failure. However, mis-segmentations can be easily verified by overlaying the segmented image on top of the original MRA dataset.

Our system can easily correct under-segmentations. We do this by identifying a seed point in the missed region and running the algorithm to segment this missed region separately. A different merging threshold is often required. The original segmentation can then be combined with the missed region segmented separately to give the final segmented atrium. In a similar way, possible over-segmentations can also be corrected by identifying a seed point within the over-segmented region and running the algorithm to subtract this region. We have found the subdivision merging process leaking into the pulmonary artery in some instances thus causing the over-segmentation. In such cases, a seed point selected inside the pulmonary artery and running the algorithm with a new merging threshold value allows the over-segmented artery to be segmented and removed from the final segmentation. It is also important to select the right value for the merging threshold (equation 1). Selecting a slightly higher threshold than what is appropriate can easily lead to over-segmentation and cause the merging process to leak into neighbouring structures. There are yet no means of automatically selecting this threshold value, and we currently rely on a trial-anderror approach.

\section{CONCLUSIONS}

We have presented a technique for automatically segmenting the left atrium from MRA datasets and a technique for segmenting the atrial body using level set methods. The atrium segmentation technique exploits the fact that partial volume affected voxels in MRA can cause the atrial blood pool to become connected to the blood pool of neighbouring structures via narrow regions. The Euclidean distance transformation of the blood pool is produced and is used to schematically subdivide the image into disjoint subdivisions. These subdivisions are later merged automatically starting from a seed point and stopping at the partial volume affected narrowings, yielding the segmented left atrium. In image-guided RFCA, detection and quantification of pulmonary venous drainage and ostium are important. Segmentation of the atrial body is a first step before the pulmonary vessels can be detected and labelled. Ostium locations can then be easily identified to lie on the separating surface between an atrial body and a pulmonary venous drainage. Our second technique for segmenting the atrial body relies on evolving interfaces modelled using level sets. Imposing the correct propagating constraints and choosing a good speed function enables the propagating front to stay within the atrial body and does not allow it to encroach into pulmonary vessels.

The system has been tested successfully on 20 diverse MRA patient datasets and the segmentation results have been validated qualitatively by an expert clinician. In future research, we will present our results on a larger collection of MRA datasets and also include a quantitative means for validating the segmentation results. Future research directions include automatic detection of pulmonary veins and a quantification of ostium. Automatic labelling of pulmonary vessels can also be very useful in classifying an atrium's anatomy.

\section{REFERENCES}

[1] Atrial Fibrillation Investigators, "Risk factors for stroke and efficacy of antithrombotic therapy in atrial fibrillation: analysis of pooled data from five randomized controlled trials," Archives of Internal Medicine 154(13), 1449-1457 (1994).

[2] Lesh, M. D., Van Hare, G. F., Epstein, L. M., et. al. "Radiofrequency Catheter Ablation of Atrial Arrhythmias: Results and Mechanisms," American Heart Association 89(3), 1075-1089 (1994). 
[3] Marom, E. M., Herndon, J. E., Kim, Y. H., et. al. "Variations in Pulmonary Venous Drainage to the Left Atrium: Implications for Radiofrequency Ablation,” Radiology 230(3), 824-829 (2004).

[4] Berg, J. V., and Lorenz, C., "Accurate left atrium segmentation in multislice CT images using a shape model," Proc. of the SPIE 5747, 351-360 (2005).

[5] John, M., and Rahn, N., "Automatic-left atrium segmentation by cutting the blood pool at narrowings," Proc. of MICCAI, 798-805 (2005).

[6] Otsu, N., "A threshold selection method from gray-level histogram," IEEE Tran. on Systems, Man. and Cybernet 9(1), 62-66 (1978).

[7] Maurer, C. R., Qi, R., Raghavan, V., “A linear time algorithm for computing exact euclidean distance transforms of binary images in arbitrary dimensions," IEEE Trans. on Patt. Analysis and Mach. Intell. 25(2), 265-270 (2003).

[8] Haissauguerre, M., Jais, P., Shah, D. C., et. al. "Spontaneous initiation of AF by ectopic beats originating in the pulmonary veins" The New Eng. Jour. of Med. 339(10), 659-666 (1998).

[9] Nakashima, H., Kumagai, K., Noguchi, H., et. al. "Evaluation of the recurrence of atrial fibrillation after pulmonary venous ablation" Jap. Coll. of Cardio. 40(3), 87-94 (2002).

[10] Iesaka, A., "Complications of catheter ablation of atrial fibrillation: cause, prevention and management," Journal of Cardiovascular Electrophysiology, 17(12), 50-55 ( 2007).

[11] Sethian, J. A., "Curvature and evolution of fronts," Comm. In Math. Phys., 101, 487-499 (1985).

[12] Malladi, R., Sethian, J., Vemuri, C. B., "Shape modeling with front propagation: a level set approach", IEEE Trans. on Pattern Analysis and Machine Intelligence, 17(2), 158-175 (1995).

[13] Sethian, J. A., "Numerical algorithms for propagating interfaces: Hamilton-Jacobi equations and conservation laws", Jour. of Diff. Geom. 31(1), 131-161 (1990). 